\title{
Could strain echocardiography help to assess systolic function in critically ill COVID-19 patients?
}

\author{
Filipe Gonzalez ${ }^{1} \cdot$ Rui Gomes $^{1}$ · Jacobo Bacariza ${ }^{1} \cdot$ Frederic Michard $^{2}(\mathbb{C}$
}

Received: 28 January 2021 / Accepted: 15 February 2021 / Published online: 27 February 2021

(c) The Author(s), under exclusive licence to Springer Nature B.V. part of Springer Nature 2021

\begin{abstract}
Strain echocardiography enables the automatic quantification of the global longitudinal strain (GLS), which is a direct measure of ventricular shortening during systole. In the current context of overwhelmed ICUs and clinician shortage, GLS has the advantage to be quick and easy to measure by non-experts. However, little is known regarding its value to assess bi-ventricular systolic function in critically ill COVID-19 patients. Therefore, we designed a study to compare right and left ventricular GLS with classic echo-Doppler indices of systolic function, namely the ejection fraction for the left ventricle (LVEF) and the fractional area change (FAC), the tricuspid annular plane systolic excursion (TAPSE), and the tissue Doppler velocity of the basal free lateral wall (S') for the right ventricle. Eighty transthoracic echocardiographic evaluations done in 30 ICU patients with COVID-19 were analyzed. We observed a fair relationship $(r=0.73, p<0.01)$ between LVEF and left ventricular GLS. The GLS cut-off value of $-22 \%$ identified a LVEF $<50 \%$ with a sensitivity of $63 \%$ and a specificity of $80 \%$. All patients with a GLS $>-17 \%$ had a LVEF $<50 \%$. Although statistically significant, relationships between FAC $(r=0.41$, $\mathrm{p}<0.01)$, TAPSE $(\mathrm{r}=0.26, \mathrm{p}<0.05)$ and right ventricular GLS were weak. S' was not correlated with right ventricular GLS. In conclusion, left ventricular GLS was useful to assess left ventricular systolic function. However, right ventricular GLS was poorly correlated with FAC, TAPSE and S'. Further studies are needed to clarify what is the best method to assess right ventricular systolic function in ICU patients with COVID-19.
\end{abstract}

Keywords COVID-19 Echocardiography $\cdot$ Speckle tracking $\cdot$ Global longitudinal strain $\cdot$ Systolic function $\cdot$ Point-of-care ultrasound

$\begin{array}{ll}\text { Abbreviations } \\ \text { FAC } & \text { Fractional area change } \\ \text { GLS } & \text { Global longitudinal strain } \\ \text { ICU } & \text { Intensive care unit } \\ \text { LVEF } & \text { Left ventricular ejection fraction } \\ \text { POCUS } & \text { Point of care ultrasound } \\ \text { S' } & \text { Tissue Doppler velocity of the basal free lateral } \\ & \text { wall }\end{array}$

TAPSE Tricuspid annular plane systolic excursion

Agency for Clinical Research and Biomedical Innovation (AICB), Portugal, \#BI12351134

Frederic Michard

frederic.michard@bluewin.ch

1 Intensive Care Department, Hospital Garcia de Orta, Almada, Portugal

2 MiCo, Consulting \& Research, Denens, Switzerland
Speckle tracking or strain echocardiography enables the measurement of the global longitudinal strain (GLS) of right and left ventricles. The GLS is a quantification of myocardial longitudinal shortening during systole [1,2]. GLS values are expressed as a negative percentage (shortening) and usually range between -20 and $-30 \%$ for both ventricles. Less negative values (e.g. $-15 \%$ ) indicate a decrease in longitudinal shortening [1,2]. The GLS is automatically calculated by modern echocardiographic software from a single cardiac view (Fig. 1). In this respect, the estimation of GLS has been shown to be quicker and less operator-dependent than the estimation of classic echo-Doppler indices of ventricular function [3, 4]. It may therefore boost the adoption and improve the accuracy of Point Of Care UltraSound (POCUS) evaluations which, in surgical and critically ill patients, are increasingly done by non-cardiologists $[5,6]$.

In COVID-19 patients, a limited number of echocardiographic evaluations have been done with speckle tracking [7-9]. These evaluations have reported a frequent decrease in right and left ventricular GLS, but they did 
Fig. 1 Top: Example of left ventricular (LV) global longitudinal strain (GLS) echocardiographic image and measurement. Bottom: Correlation between LV ejection fraction (LVEF) and LV GLS
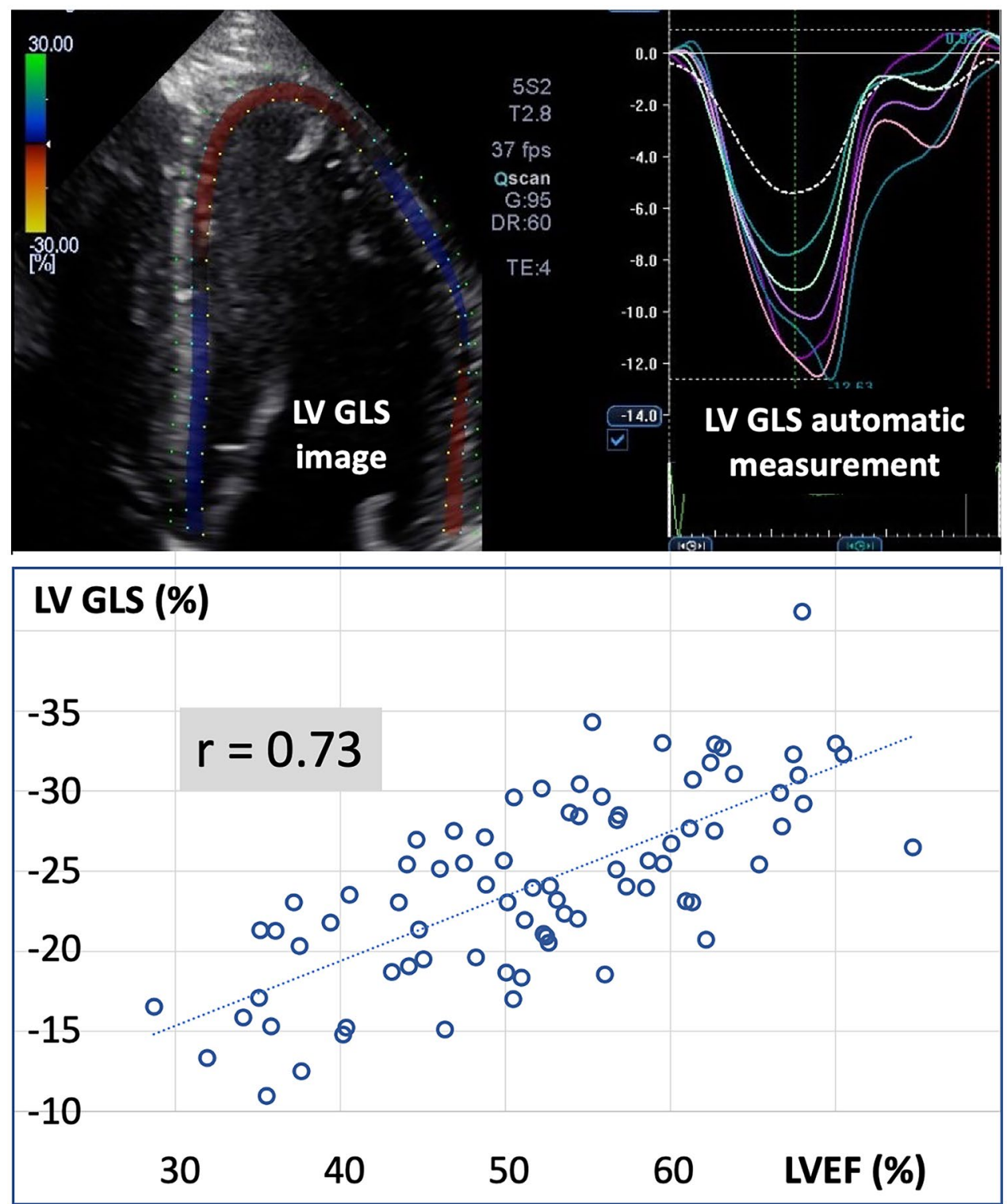

not provide any comparison with classic indices of systolic function. In addition, studies published so far have not been done in Intensive Care Unit (ICU) patients. As a result, little is known regarding right and left ventricular GLS in the most severe COVID-19 cases.

The present study was designed to compare right and left ventricular GLS measurements to classic echo-Doppler indices of bi-ventricular systolic function in ICU patients with COVID-19. We hypothesized that GLS measurements are more reproducible than classic measurements, and provide, if not interchangeable, at least consistent information regarding the systolic function of both ventricles.

\section{Methods}

The study was approved by the ethical committee of hospital Garcia de Orta, Almada, Portugal (\#BI12351134, Approval date 05/07/2020), and written informed consent was obtained for all patients. Transthoracic echocardiographic evaluations were performed in COVID-19 patients admitted in the ICU of Hospital Garcia de Orta. All adult patients admitted between June and September 2020 were screened for eligibility. Patients admitted for acute myocardial infarction or pulmonary embolism with a positive COVID-19 Polymerase Chain 
Reaction (PCR) swab test were excluded from the study, as well as patients in whom good quality transthoracic images were impossible to obtain. For all remaining COVID-19 patients, we performed an echocardiography the day of admission, then at day 3 and day 7 . All ultrasound recordings and measurements were done by skilled operators and reviewed by one of us (FG) who holds the European Diploma in Advanced Critical Care Echocardiography. A 2D wall motion tracking software (Xario 200, Canon Medical Systems, Otawara, Japan) was used to assess right and left ventricular GLS from a 4-cavity apical view. The endocardial border was automatically detected using 3 reference points (basal septum, basal free wall and apex) and manually adjusted, if deemed necessary, by the operator. Then, the software automatically performed speckle tracking on a frame-to-frame basis. Traditional indices of systolic function were also assessed, namely the ejection fraction for the left ventricle (LVEF) and the fractional area change (FAC), the tricuspid annular plane systolic excursion (TAPSE), and the tissue Doppler velocity of the basal free lateral wall (S') for the right ventricle [10].

For each index, 3 consecutive measurements were performed and averaged. The reproducibility was calculated as the standard deviation divided by the mean (coefficient of variation). Results were expressed as mean \pm SD or median (IQR) when appropriate and reproducibilities were compared using a non-parametric Mann \& Whitney test. A p value $<0.05$ was considered statistically significant.

\section{Results}

Forty COVID-19 patients were screened, and 10 were excluded because of poor echogenicity $(n=7)$, pulmonary embolism $(\mathrm{n}=2)$ and myocardial infarction $(\mathrm{n}=1)$. Among the 30 remaining patients, 7 were discharged and 3 died before $\mathrm{J} 7$. Therefore, a total of 80 echocardiographic evaluations done in 30 patients were available for analysis. Main patients' characteristics and outcomes are presented in Table 1. Twenty-one echocardiographic evaluations were done during mechanical ventilation, 11 during vasopressor support and 3 during inotropic support. Echocardiographic indices of bi-ventricular systolic function and reproducibility of measurements are presented in Table 2.

We observed a fair relationship $(r=0.73, p<0.01)$ between LVEF and left ventricular GLS (Fig. 1). The GLS cut-off value of $-22 \%$ identified a LVEF $<50 \%$ with a sensitivity of $63 \%$ and a specificity of $80 \%$. All patients with a GLS $>-17 \%$ had a LVEF $<50 \%$ (Fig. 1).

Although statistically significant, relationships between FAC $(r=0.41, p<0.01)$, TAPSE $(r=0.26, p<0.05)$ and right ventricular GLS were weak (Fig. 2). S' was not correlated with right ventricular GLS. A TAPSE value $<17 \mathrm{~mm}$
Table 1 Characteristics at ICU admission and outcome of the study population

\begin{tabular}{ll}
\hline Gender (F/M) & $11 / 19$ \\
\hline Age (year) & $61 \pm 15$ \\
Body Mass Index $\left(\mathrm{kg} / \mathrm{m}^{2}\right)$ & $31 \pm 6$ \\
Chronic hypertension, $\mathrm{n}(\%)$ & $22(73 \%)$ \\
Diabetes, n (\%) & $9(30 \%)$ \\
Chronic obstructive pulmonary disease, n (\%) & $3(10 \%)$ \\
$\mathrm{Days} \mathrm{since} \mathrm{onset} \mathrm{of} \mathrm{symptoms,} \mathrm{median} \mathrm{(IQR)}_{\mathrm{PaO}_{2} / \mathrm{FiO}}$ ratio & $8(5-10)$ \\
$\mathrm{Treatment}$ with Remdesivir & $177 \pm 65$ \\
$\mathrm{Mechanical} \mathrm{ventilation,} \mathrm{n} \mathrm{( \% )}_{\text {Median ICU length of stay, days (IQR) }}$ & $70(33 \%)$ \\
Median Hospital length of stay, days (IQR) & $7(23 \%)$ \\
ICU mortality, n (\%) & $8(5-12)$ \\
\hline
\end{tabular}

Table 2 Strain and classic echocardiographic indices of systolic function

\begin{tabular}{llll}
\hline $\begin{array}{l}\text { Systolic function } \\
\text { indices }\end{array}$ & Mean \pm SD & $\begin{array}{l}\text { Range } \\
\text { Min to Max }\end{array}$ & $\begin{array}{l}\text { Reproducibility } \\
\%\end{array}$ \\
\hline $\begin{array}{l}\text { Left ventricle } \\
\text { GLS (\%) }\end{array}$ & $-24 \pm 6$ & -11 to -41 & $4.9 \pm 2.1$ \\
LVEF (\%) & $53 \pm 10$ & 29 to 75 & $4.8 \pm 2.1$ \\
Right ventricle & & & \\
GLS (\%) & $-26 \pm 8$ & -10 to -55 & $4.9 \pm 2.1$ \\
FAC (\%) & $44 \pm 11$ & 23 to 65 & $4.9 \pm 2.1$ \\
TAPSE (mm) & $22 \pm 3$ & 15 to 32 & $4.1 \pm 1.4$ \\
S' $(\mathrm{cm} / \mathrm{s})$ & $15 \pm 3$ & 8 to 25 & $4.9 \pm 2.1$
\end{tabular}

$G L S$ global longitudinal strain, $L V E F$ global ejection fraction, FAC fractional area change, TAPSE tricuspid annular plane systolic excursion, $S$ ' tissue Doppler velocity of the basal free lateral wall

and a $S^{\prime}$ value $<10 \mathrm{~cm} / \mathrm{s}$ were observed during only $4 / 80$ (5\%) echocardiographic evaluations, whereas a FAC $<35 \%$ and a right ventricular GLS $>-20 \%$ were observed during 18 (23\%) and $23(29 \%)$ evaluations, respectively.

\section{Discussion}

Multiple factors may impact cardiac function in critically ill COVID-19 patients, including systemic inflammation, hypoxemia, myocardial ischemia, myocarditis, mechanical ventilation with positive end-expiratory pressure and pulmonary embolism [11-13]. A quick POCUS cardiac evaluation is therefore highly desirable in this context, but a surge of acutely ill patients may limit the feasibility of an operatordependent and time-consuming procedure [14]. Recent studies suggest that a decrease in right and left ventricular GLS is an independent predictive factor of tracheal intubation 
Fig. 2 Left: Example of right ventricular (RV) global longitudinal strain (GLS) echocardiographic image. Right: Correlations between the right ventricular fractional area change (FAC), the tricuspid annular plane systolic excursion (TAPSE) and RV GLS
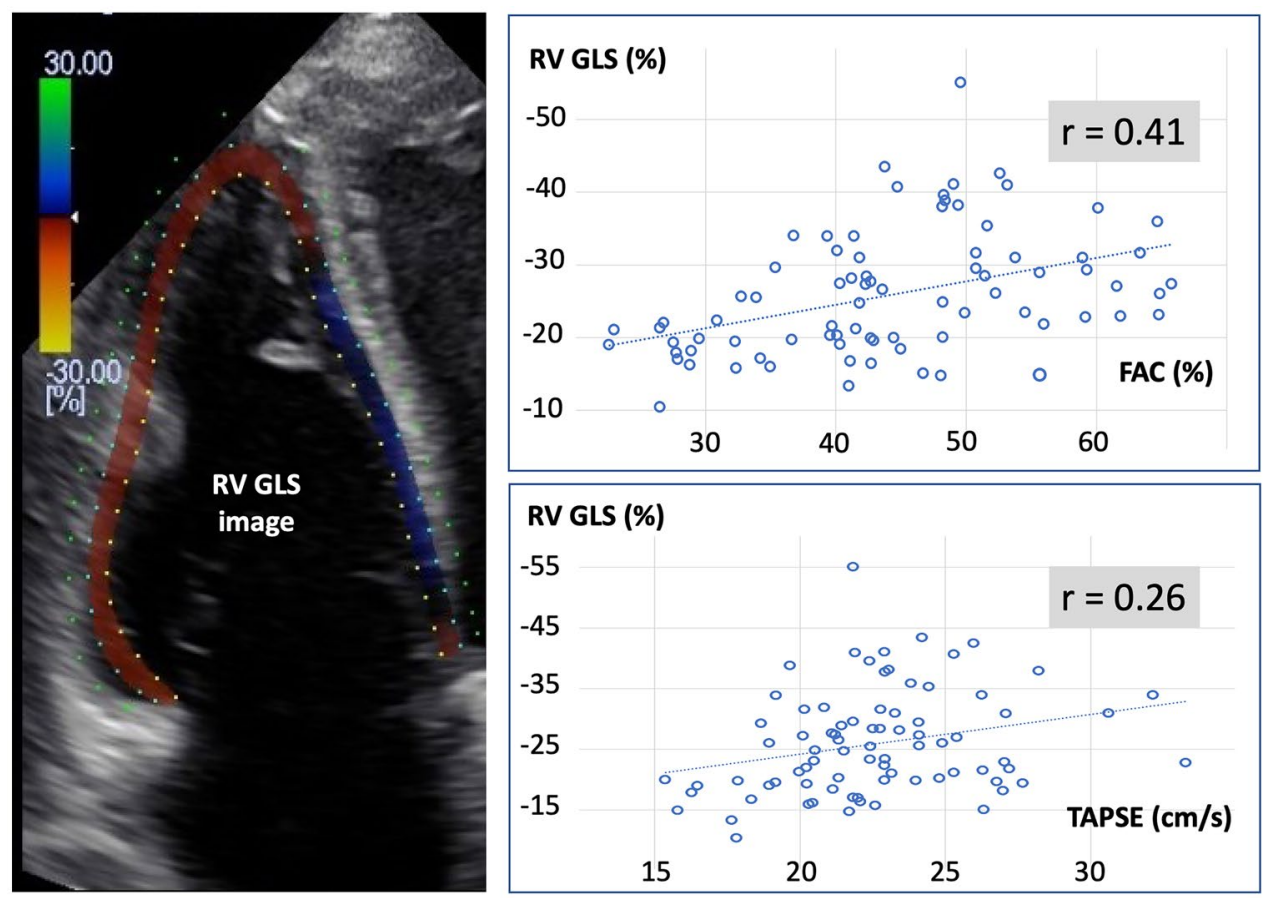

and in-hospital mortality in COVID-19 patients [7, 8]. The ability to quickly assess both right and left ventricular function with strain echocardiography, regardless of the operator training level $[3,4]$, is therefore very appealing in this context.

Our findings suggest that left ventricular GLS may provide actionable information about left ventricular systolic function in ICU patients with COVID-19. They are consistent with the results of previous reports done in cardiac patients [4] and with a recent meta-analysis of studies done in critically ill patients with severe sepsis and septic shock [2] suggesting that left ventricular GLS is a reliable marker of left ventricular systolic function. The GLS is a direct measure of myocardial shortening. In contrast, the LVEF is calculated from the end-systolic and end-diastolic volumes. In addition, the geometric assumptions made by the disc Simpson's method may not hold true for all patients [2]. The LVEF is therefore an indirect measure of systolic function. When the GLS was $>-17 \%$, the LVEF was always $<50 \%$. Conversely, when the LVEF was $<50 \%$ the GLS was $<-20 \%$ during 16 evaluations (20\%), and $<-25 \%$ during 7 evaluations (9\%). Therefore, although significantly correlated, the two indices were not interchangeable, and the lack of reference method does not allow us to conclude which one was the best marker of left ventricular systolic function. Finally, the reproducibility of measurements was not better for left ventricular GLS than for LVEF.

The right ventricular GLS was poorly correlated with classic indices of right ventricular systolic function and the intra-operator reproducibility of GLS measurements was not better. We observed a significant discrepancy between the information provided by TAPSE and S' on one hand, and by FAC and right ventricular GLS on the other hand. Indeed, TAPSE and S' were abnormally low in only $5 \%$ of our evaluations, whereas FAC and right ventricular GLS were abnormal during around 1/4 of the evaluations. TAPSE is widely used to assess right ventricular systolic function [10]. However, both in chronic heart failure and cardiac surgical patients, TAPSE has been shown to be less sensitive to detect right ventricular dysfunction and less accurate to predict outcome than right ventricular GLS [15, 16]. In addition, in a recent study done in 282 critically ill patients with septic shock, TAPSE was unable to discriminate patients with or without right ventricular failure [17]. The lack of sensitivity of TAPSE and S' to detect systolic dysfunction may be related to the fact that they only represent a small portion of the right ventricle (regional assessment of systolic function), as well as to the angle dependency of their measurements $[18,19]$. The FAC is calculated from the endsystolic and end-diastolic area measurements and, therefore, like the LVEF, is an indirect measure of systolic function. In contrast, the right ventricular GLS is a direct measure of myocardial shortening. However, it includes the interventricular septum shortening and is therefore influenced by left ventricular systolic function [20]. Whether a better correlation with FAC could be observed when restricting strain measurements to the right ventricular free wall remains to be determined.

Our study has several limitations. First, GLS measurements have been shown to be software dependent [19], so 
that we cannot exclude that our findings may have been different if we had used a different commercially available speckle tracking software. Second, echocardiographic evaluations were performed by experienced operators, which may explain why the intra-observer reproducibility of measurements was as good for classic indices than for GLS measurements. Further studies would be useful to investigate whether the reproducibility might be better for strain than for classic measurements when evaluations are done by trainees or echo beginners. Finally, several studies have suggested that the right ventricular GLS is a better marker of systolic dysfunction than the FAC [16] or even than the 3D right ventricular ejection fraction [1]. In our study, the lack of gold standard method, such as magnetic resonance imaging [1], does not allow us to draw any definitive conclusion regarding the respective value of GLS and FAC to assess right ventricular systolic function.

\section{Conclusion}

Our study is the first comparison between strain and classic echocardiographic indices of bi-ventricular systolic function in critically ill COVID-19 patients. Our findings suggest that, although not interchangeable, left ventricular GLS and LVEF provide consistent information regarding left ventricular systolic function. In the current context of overwhelmed ICUs and clinician shortage, the left ventricular GLS has the advantage to be quick and easy to measure by non-experts. The right ventricular GLS was weakly correlated with FAC, poorly with TAPSE, and was not correlated in any way with S'. Further studies are needed to clarify whether FAC or right ventricular GLS may preferentially be used for the assessment of right ventricular systolic function in ICU patients with COVID-19. Finally, the reproducibility of measurements was not better for GLS than for classic indices.

Acknowledgements The authors thank Pedro Pereira from Canon, Portugal, for providing the 2D Wall Motion Tracking software; Hugo Michard, Switzerland, for data analysis; and Pr Thierry Fumeaux, University of Geneva, Switzerland, national expert in POCUS, for reviewing the final version of the manuscript.

Author contributions FG had full access to all of the data in the study and takes responsibility for the integrity of the data and the accuracy of the data analysis. FG, RG, JB and FM contributed substantially to the study design, data analysis and interpretation, and the writing of the manuscript.

Funding Foundation for Science and Technology (FTC), Portugal.

\section{Compliance with ethical standards}

Conflict of interest FM is the founder and managing director of MiCo, a Swiss consulting and research firm. MiCo does not sell any medical products and FM does not own any shares from any MedTech company. FG, RG and JB have nothing to declare.

Ethical approval The study was approved by the ethical committee of hospital Garcia de Orta, Almada, Portugal (\#BI12351134, Approval date $05 / 07 / 2020$ ).

Consent to participate Written informed consent was obtained for all patients.

\section{References}

1. Lu KJ, Chen JXC, Profitis K, et al. Right ventricular global longitudinal strain is an independent predictor of right ventricular function: a multimodality study of cardiac magnetic resonance imaging, real time three-dimensional echocardiography and speckle tracking echocardiography. Echocardiography. 2015;32:966-74.

2. Sanfilippo F, Corredor C, Fletcher N, et al. Left ventricular systolic function evaluated by strain echocardiography and relationship with mortality in patients with severe sepsis or septic shock: a systematic review and meta-analysis. Crit Care. 2018;22:183.

3. Karlsen S, Dahlslett T, Grenne B, et al. Global longitudinal strain is a more reproducible measure of left ventricular function than ejection fraction regardless of echocardiographic training. Cardiovasc Ultrasound. 2019;17:18.

4. Benyounes N, Lang S, Soulat-Dufour L, et al. Can global longitudinal strain predict reduced left ventricular ejection fraction in daily echocardiographic practice? Arch Cardiovasc Dis. 2015;108:50-6.

5. Trauzeddel RF, Ertmer M, Nordine M, et al. Perioperative echocardiography-guided hemodynamic therapy in high-risk patients: a practical expert approach of hemodynamically focused echocardiography. J Clin Monit Comput. 2020. https://doi.org/10.1007/ s10877-020-00534-7.

6. Vieillard-Baron A, Millington SJ, Sanfilippo F, et al. A decade of progress in critical care echocardiography: a narrative review. Intensive Care Med. 2019;45:770-88.

7. Rothschild E, Baruch G, Szekely Y, et al. The predictive role of left and right ventricular speckle-tracking echocardiography in COVID-19. JACC Cardiovasc Imag. 2020;13:2287-99.

8. Baycan OF, Barman HA, Atici A, et al. Evaluation of biventricular function in patients with COVID-19 using speckle tracking echocardiography. Int J Cardiovasc Imaging. 2020. https://doi. org/10.1007/s10554-020-01968-5.

9. Croft LB, Krishnamoorthy P, Ro R, et al. Abnormal left ventricular global longitudinal strain by speckle tracking echocardiography in COVID-19 patients. Fut Cardiol. 2020. https://doi. org/10.2217/fca-2020-0121.

10. Schneider M, Aschauer S, Mascherbauer J, et al. Echocardiographic assessment of right ventricular function: current clinical practice. Int J Cardiovasc Imaging. 2019;35:49-56.

11. Michard F, Vieillard-Baron A. Critically ill patients with COVID19: are they hemodynamically unstable and do we know why? Intensive Care Med. 2021. https://doi.org/10.1007/s00134-02006238-5

12. Szekely Y, Lichter Y, Taieb P, et al. Spectrum of cardiac manifestations in COVID-19. A systematic echocardiographic study. Circulation. 2020;142:342-53. 
13. Dweck MR, Bularga A, Hahn RT, et al. Global evaluation of echocardiography in patients with COVID-19. Eur Heart J Cardiovasc Imaging. 2020;21:949-58.

14. Michard F, Malbrain ML, Martin G, et al. Haemodynamic monitoring and management in COVID-19 intensive care patients: an international survey. Anesth Crit Care Pain Med. 2020;39:563-9.

15. Carluccio E, Biagioli P, Alunni G, et al. Prognostic value of right ventricular dysfunction in heart failure with reduced ejection fraction: Superiority of longitudinal strain over tricuspid annular plane systolic excursion. Circ Cardiovasc Imaging. 2018;11:e006894.

16. Ternacle J, Berry M, Cognet T, et al. Prognostic value of right ventricular two-dimensional global strain in patients referred for cardiac surgery. J Am Soc Echocardiogr. 2013;26:721-6.

17. Vieillard-Baron A, Prigent A, Repessé X, et al. Right ventricular failure in septic shock: characterization, incidence and impact on fluid responsiveness. Crit Care. 2020;24:630.

18. Bootsma IT, Scheeren TWL, de Lange F, et al. The reduction in right ventricular longitudinal contraction parameters is not accompanied by a reduction in general right ventricular performance during aortic valve replacement: an explorative study. J Cardiothorac Vasc Anesth. 2020;34:2140-7.

19. Rudski LG, Lai W, Afilalo J, et al. Guidelines for the echocardiographic assessment of the right heart in adults: a report from the American Society of Echocardiography. J Am Soc Echocardiogr. 2010;23:685-713.

20. Carluccio E, Biagioli P, Lauciello R, et al. Superior prognostic value of right ventricular free wall compared to global longitudinal strain in patients with heart failure. J Am Soc Echocardiogr. 2019;32:836-44.

Publisher's Note Springer Nature remains neutral with regard to jurisdictional claims in published maps and institutional affiliations. 